4.

Tierethik im Kontext systemischer Herausforderungen 


\title{
Tierethik im Spannungsfeld von Tierwohl, Landwirtschaft und Agrarpolitik
}

\author{
Markus Vogt
}

\section{Systemische Herausforderungen und das Design der Fragestellung}

Im gesellschaftlichen Umgang mit Tieren gibt es gegenwärtig vor allem zwei grundlegende Herausforderungen, die beide vorrangig mit der Landnutzung und damit der Agrarpolitik zusammenhängen: zum einen die offensichtliche Diskrepanz zwischen den Bedingungen der Tierhaltung in der moderneren Nahrungsmittelproduktion und dem Anspruch, die Tiere als Mitgeschöpfe in ihrem Eigenwert zu achten; zum anderen das sechste große Artensterben, das derzeit auf unserem Planeten stattfindet und das als ein mit dem Klimawandel vergleichbarer tiefer Einschnitt in der Evolution des Lebens angesehen wird (Ulrich Kutschera 2016, 57-66). Das meistdiskutierte Beispiel hierfür in jüngster Zeit ist das Insektensterben, das in der politischen Debatte um Pestizideinsatz und Biodiversität große öffentliche Aufmerksamkeit erhalten hat. Auch hier geht es um vernachlässigte Nebenwirkungen der Agrarpolitik und der landwirtschaftlichen Praxis.

Der weltweite, das menschliche Selbstverständnis berührende Animal Turn (Clemens Wustmans 2015, 167-170; Johann Ach/Dagmar Borchers [Hg.] 2018) als eine kulturwissenschaftliche, umwelthistorische und ethisch-praktische Hinwendung zu Tieren steht in einem auffallenden Widerspruch zur gleichzeitigen Zunahme der industrialisierten Landwirtschaft. Gerade in der Tierhaltung steht diese in globaler Konkurrenz unter enormem Effizienzdruck und sieht mehrheitlich kaum Möglichkeiten, den ethischen Erwartungen zu entsprechen. Man kann die gegenwärtige Situation als eine fast schizophrene Spaltung zwischen ethischem Anspruch und der gesellschaftlichen Praxis deuten. Als Konsumenten ist der überwiegende Anteil der Bevölkerung daran beteiligt. Deshalb ist das othering der moralischen Empörung mehrheitlich nicht angemessen. 


\subsection{Systemische Herausforderungen}

Die Probleme des Tierschutzes in der Landwirtschaft sind in besonderer Weise von systemischen Wirkungszusammenhängen geprägt. Das macht ihre ethische und ökosoziale Brisanz aus, zugleich aber auch ihre steuerungs- und kommunikationstheoretische Komplexität. Durch die Dominanz des pathozentrisch-individualethischen Ansatzes der Tierethik wurden diese Zusammenhänge lange unterschätzt.

Die systemischen Herausforderungen betreffen vor allem zwei Aspekte:

1. Zum einen die Handlungszwänge der verschiedenen Akteure in dem von einem hohen Grad an Komplexität, Abhängigkeiten und verschränkten Handlungsketten geprägten Agrar- und Ernährungssystem. So sehen sich die Bauern seit Jahrzehnten zwischen dem hohen Preisdruck der Lebensmittelindustrie sowie der europäisierten und globalisierten Agrarmärkte und den aus ihrer Sicht oft idealisierten ethischen Erwartungen der umweltbewegten Zivilgesellschaft eingeklemmt. Diese Handlungszwänge, die einzelne Menschen, Betriebe und Branchen erleben, sind dabei jedoch keineswegs naturgegeben, sondern gesellschaftlich erzeugt und damit sozialethisch $z u$ gestalten. In der Kommunikation ist jedoch zu beachten, dass Landwirte und Nutztierhalter in gleicher Weise „Täter" und „Opfer“ der Umwelt- und Klimaveränderungen sowie agrarpolitischer Systemzwänge sind (Antonia Weishaupt 2019, 13). Die Agrarpolitik ist eines der anschaulichsten Beispiele für eine ursprünglich durch Solidarität motivierte Fehlallokation von Mitteln mit am Ende ökologisch, ökonomisch und sozial negativen Nebenwirkungen. So gehört die Gemeinsame Agrarpolitik (GAP) zu den ältesten und finanziell umfangreichsten Politikbereichen der Europäischen Union: 1982 entfielen ca. 70 Prozent des EU-Haushalts auf die GAP, gegenwärtig immer noch 38 Prozent (Bundesministerium für Ernährung und Landwirtschaft 2019 a). ${ }^{1}$ Die Mehrheit der Landwirte käme im derzeit bestehenden System ohne EU-Förderungen finanziell nicht über die Runden. Zugleich ist die Förderung Ursache für Über- und Fehlproduktion, einen ruinösen Preiswettbewerb und Lebensmittelverschwendung (ca. ein Drittel wird weltweit weggeworfen, wobei die Ursachen sich deutlich von Region zu Region unterscheiden, z. B. fehlende Kühlketten in tropischen Ländern oder Verschwendung durch End-

1 In Deutschland stehen der Landwirtschaft jährlich 6,2 Milliarden Euro zur Verfügung: Durchschnittlich beziehen Landwirte in Deutschland 40 Prozent ihres Einkommens aus Subventionen (37 Prozent im EU-Durchschnitt). 
verbraucherinnen und Endverbraucher in Industrieländern). Mit der Ernährung von 7,6 Mrd. Menschen erbringt das Agrarsystem eine Höchstleistung; zugleich leidet es unter enormen Fehlsteuerungen auf Kosten zahlloser Menschen, Tiere und Naturräume. Tierethik muss nüchtern die Handlungszwänge im Spannungsfeld von Tierwohl, Landwirtschaft und Agrarpolitik zur Kenntnis nehmen, ohne vor ihnen zu resignieren.

2. Das zweite systemische Problem der Landwirtschaft sind die komplexen ökologischen Auswirkungen. Ein Gutachten des Weltklimarats (IPCC) rechnet aus, dass derzeit (2019) ca. 23 Prozent der Treibhausgasemissionen aus der Landwirtschaft bzw. der veränderten Landnutzung stammen (Simon Michel-Berger 2019b). Tierhaltung ist ein treibender Faktor der gegenwärtigen Umweltprobleme (Antonia Weishaupt 2019, 19-27). Seit Jahren weisen die Daten zur Erdsystemforschung auf der Basis des Planetary-Boundaries-Konzeptes im Landwirtschaftsbereich die markantesten „roten Ampeln“ auf: Der hohe Dünger- und Pestizideinsatz belastet den Wasserhaushalt und die Böden; die Futtermittel- und Biospritproduktion sind der treibende Faktor für die Rodung der Regenwälder, die als „das grüne Herz des Planeten“ unverzichtbar sind; die Änderung der Landnutzung reduziert Lebensräume wilder Tiere und führt derzeit zum sechsten großen Artensterben in der Geschichte der Evolution des Lebens (Will Steffen et al. 2015). Fernab unserer Wahrnehmung ereignet sich aufgrund der primär durch expansive Landnutzung verursachten Zerstörung von Lebensräumen ein Massensterben von Tierarten, das mindestens zehn bis hundert Mal schneller als im Durchschnitt der letzten zehn Millionen Jahre stattfindet und sich stetig beschleunigt (Ulrich Kutschera 2016, 57-66; Helmholtz-Zentrum für Umweltforschung [Hg.] 2019, 9). Zum großen Teil handelt es sich um unbekannte Arten oder solche, die unsere Emotionen wenig ansprechen, die jedoch für die Evolutionsfähigkeit des Lebens auf der Erde von grundlegender Bedeutung sind. Auch wenn es hier schwierig ist, systemische Kipppunkte quantitativ zu prognostizieren, gibt es zahlreiche Indizien dafür, dass die massive Reduktion der Biodiversität nicht lange mit dem Erhalt der uns unbekannten Strukturen der Ökosysteme vereinbar sein wird. Biodiversität ist neben der Energieversorgung der zweite, bisher weit unterschätzte Brennpunkt des Ökozids, auf den unsere Zivilisation derzeit zuzusteuern scheint. Die massive Zunahme der Nutztierhaltung hat darin eine systemische Schlüsselstellung. 


\subsection{Zur Verortung der Tierschutzethik im Kontext der Agrarpolitik}

Vor diesem Hintergrund stellen sich mit der Agrarpolitik sozialethische Grundfragen nach der gerechten und effizienten Steuerung komplexer Systeme sowie nach der Verortung in umwelt-, tier- und gesellschaftsethischen Kontexten. In Deutschland ist die Tierethik politisch nicht beim Umweltministerium, sondern primär beim Landwirtschaftsministerium angesiedelt. Damit ist jedoch eine spezifische Einengung der Fragestellung verbunden: Kriterien des Tierschutzes werden schon aufgrund dieses Kontextes als Modifikationen der bestehenden Praxis gedacht und zu einem auf halbherzige Kompromisse angelegten Begleitdiskurs verflacht. Die zentrale Bedeutung der Nutztierhaltung für Umwelt- und Klimaveränderungen wird aufgrund der Nichtzuständigkeit des Umweltministeriums politisch bisher massiv vernachlässigt (Antonia Weishaupt 2019, 15).

Die Verortung der Tierethik hat vor diesem Hintergrund nicht nur eine theoriestrategische Bedeutung, sondern führt auch zu unterschiedlichen inhaltlichen Akzentsetzungen: Im Kontext der Landwirtschaft stehen pragmatische Kriterien der Leidvermeidung sowie gesellschaftspolitische Fragen im Mittelpunkt. Ernährungsethik beschäftigt sich mit der Rechtfertigungsfähigkeit der Tötung von Tieren durch Menschen. Umweltethische Zugänge fokussieren auf ökologische Auswirkungen der Massentierhaltung. Konzipiert man die Tierethik als eigenständiges Reflexionsfeld, wird vorrangig der personenanaloge Status von Tieren diskutiert, was meist in Grundsatzdebatten mündet. Darüber hinaus ist damit ein individualethischer Akzent verbunden. So geht beispielsweise Nida-Rümelin davon aus, dass Tierethik primär den Umgang mit Tierindividuen thematisiere und daher aus der Umweltethik ausgegliedert werden könne (Julian Nida-Rümelin 2005, 516). ${ }^{2}$ Kriterien der Arterhaltung, die aus meiner Sicht gerade für agrarpolitische Kontexte der Tierethik ethisch nicht zu vernachlässigen sind, kommen in einem solchen Ansatz kaum in den Blick. Eine sozialethische Reflexion hat aus meiner Sicht die Aufgabe, die Stärken, Schwächen und Anschlussstellen dieser unterschiedlichen Perspektiven verständlich zu machen und eine ausgewogene Urteilsbildung zu fördern.

Ein Ort hierfür ist beispielsweise die Tierschutzkommission des Bundes, die seit 1987 durch das deutsche Bundeslandwirtschaftsministerium einbe-

2 Eher aus pragmatischen Gründen, um die Fülle an Themen zu bewältigen, haben auch Markus Vogt, Jochen Ostheimer und Frank Uekötter die Tierethik aus der Umweltethik ausgelagert (vgl. Markus Vogt et al. 2013; zur Konzeption und Abgrenzung der Umweltethik vgl. die Einführung der Herausgeber, ebd., 11-17). 
rufen wird. Sie wird zu allen Verordnungen und allgemeinen Verwaltungsvorschriften nach dem Tierschutzgesetz angehört und kann auch in Eigeninitiative zu Tierschutzfragen Stellung gegenüber dem Ministerium nehmen. Derzeit kommen von den zwölf Mitgliedern fünf aus der Forschung, vier von Tierschutzorganisationen und jeweils einer von der Firma Merck, dem Deutschen Bauernverband und der Katholischen LandvolkBewegung. Auch wenn sich die Tierethik im Kontext der Landwirtschaft nicht selten halbherzigen Kompromissen hingibt, kann man doch zugleich auch spezifische Stärken dieses Zugangs ausmachen: Er setzt sich verstärkt mit praktischen Fragen auseinander, wie Tierschutz in unserer Gesellschaft konsensual definiert und durchgesetzt werden kann. Er ist auf einen akteursorientierten Ansatz angelegt, der danach fragt, welche Verantwortung den unterschiedlichen Gruppen und Institutionen in Gesellschaft, Landwirtschaft, Ernährungsindustrie, Politik und nicht zuletzt auch in den Kirchen zukommt.

\subsection{Die Gleichzeitigkeit von Empathie und systemischer Entfremdung}

Durch den enormen Druck zur Produktionssteigerung und zunehmende Internationalisierung der Märkte steckt die Nutztierhaltung trotz des verbesserten Rechtsschutzes für Tiere in einer tiefen ethischen Krise:

„Die gesamte landwirtschaftliche Tierhaltung ist einem extremen ökonomischen Verwertungsdruck ausgesetzt, der bereits globalisiert ist. Die problematischen Methoden sind Teil einer Intensivtiernutzung, bei der das einzelne Tier unter dem Gesichtspunkt der Erzielung maximaler Leistungen in immer kürzerer Zeit betrachtet wird" (Evangelische Kirche in Deutschland/Deutsche Bischofskonferenz 2003, Nr. 15).

Aufgrund der ökonomisch-technischen Systemlogik hinkt die Praxis des Tierschutzes den ethischen und rechtlichen Vorgaben weit hinterher. Besonders am Pranger der Öffentlichkeit steht die Massentierhaltung bei Geflügel, Schweinen und Rindern in Ställen (Manfred Röhrs/Hans Sambraus 1998, 546).

Tierethik im Kontext der Agrarpolitik sieht sich vor diesem Hintergrund mit grundlegenden Spannungen und widersprüchlichen Erwartungen konfrontiert: Auf der einen Seite stehen Tierschützer mit teilweise radikalen Forderungen, idealistischen Vorstellungen oder auch militanten Strategien, aber auch mit zunehmendem empirischem Wissen über kognitive Fähigkeiten von Tieren und existenzielle Bedrohungen ihrer Lebensräume. Auf der anderen Seite stehen die auf Nutzen und Gewinn angewiese- 
nen Halter und Produzenten der Nutztiere, die in der Nachfrage nach billigen Produkten den entscheidenden Faktor sehen und damit auf die Verantwortung der Konsumenten verweisen. Die ethische Diskussion um Tierschutz ist vor diesem Hintergrund von einer tiefen Diskrepanz ganz unterschiedlicher Perspektiven, Kontexte und Bewertungen geprägt (Markus Vogt 2019, 335-339).

Martha Nussbaum sieht in der Tierethik eine grundlegende frontier of justice. Nach ihrer Bilanz sind die unübersehbaren, gravierenden Konflikte zwischen den Nutzungs- und Lebensrauminteressen der Menschen und den natürlichen Bedürfnissen der Tiere nur unvollständig auflösbar: „Wir sollten zugestehen, dass es in der Beziehung zwischen Menschen und Tieren immer einen Rest von Tragik (Hervorhebung M. V.) geben wird" (Martha Nussbaum 2010, 543).

Manche Konflikte lassen sich weder durch verantwortungsethische Abwägungen und entsprechende Rechtsverordnungen noch durch gesinnungsethische Appelle auflösen. Tierethik im Kontext der Landwirtschaft bleibt deshalb letztlich eine hybride Theorie, die bestenfalls heterogene Erfordernisse und Perspektiven in eine temporäre Balance bringen kann.

\section{Ethische Kriterien für praktischen Tierschutz}

\subsection{Das Postulat „vernünftiger Gründe“ im deutschen Tierschutzgesetz}

In der Schweiz ist der Tierschutz seit 1973 auf der Ebene der Bundesverfassung verankert (Art. 80 BV). 1992 hielt die „Würde der Kreatur“ in die Bundesverfassung Einzug (Art. 120 Abs. 2).

Das österreichische „Bundesverfassungsgesetz über die Nachhaltigkeit, den Tierschutz, den umfassenden Umweltschutz, die Sicherstellung der Wasser- und Lebensmittelversorgung und die Forschung" (BGBl. I Nr. 111/2013) formuliert lapidar: „Die Republik Österreich (Bund, Länder und Gemeinden) bekennt sich zum Tierschutz“ ( $\$ 2)$.

In Deutschland ist er seit 2002 verfassungsrechtlich verankert (Art. 20 a GG). Aufbauend auf dem Verfassungsauftrag postuliert das deutsche Tierschutzgesetz (letzte Novelle 2013), „[...] aus der Verantwortung des Menschen für das Tier als Mitgeschöpf dessen Leben und Wohlbefinden zu schützen. Niemand darf einem Tier ohne vernünftigen Grund Schmerzen, Leiden oder Schäden zufügen“ ( $\$ 1)$. 
„Ohne vernünftigen Grund“ ist ein sehr weiches Kriterium, das gegenüber den industrialisierten Verwertungsinteressen weitgehend stumpf ist. ${ }^{3}$ Angesichts der Praxis von millionenfacher Qual und Tötung von Tieren klingt der stolze Satz „Zweck dieses Gesetzes ist es, aus der Verantwortung des Menschen für das Tier als Mitgeschöpf dessen Leben und Wohlbefinden zu schützen“, wie ein Hohn (vgl. dazu die beißende Kritik von Richard David Precht 2016, 454). Im Kontext der praktischen Gesetzgebung zum Tierschutz für die Nutztierhaltung wird die Verwendung des Begriffs „Mitgeschöpf“ faktisch Teil der Legitimation einer Praxis, die seit vielen Jahren den Maximen der Maximierung von Produktivität unterliegt. Durch die Kombination mit der sehr unbestimmten Formel „Vermeidung von unnötigem und erheblichem Leid“ wurde der Begriff „Mitgeschöpf“ im deutschen Tierschutzgesetz in hohem Maße normativ stumpf.

\subsection{Brennpunkte der ethischen Debatte}

Da Schweine in ihren Bedürfnissen und Fähigkeiten hochdifferenzierte und bewegungsaktive Tiere sind, widerspricht es den Prinzipien artgerechter Tierhaltung, dass ihnen nur selten eine räumliche Strukturierung des Stalles nach Liege-, Aktivitäts-, Wühlbereich und Kotgang zuerkannt wird. Schwanzbeißen bei Schweinen ist eine weltweit bekannte Verhaltensstörung, die sowohl in konventionellen als auch alternativen Haltungsverfahren auftreten kann.

Bei Rindern ist das Grundproblem des Mangels an artgerechter Tierhaltung die Art der Fütterung: Überwiegend bekommen sie Kraftfutter, das deutlich billiger ist und die extreme Hochleistung in der Milchproduktion ermöglicht, statt Weidegras oder Heu, für das ihre Mägen eigentlich angelegt sind. So sind ihre Mägen häufig entzündet und ihre Lebenszeit ist wesentlich verkürzt.

Ein weiterer Brennpunkt der Tierethik im Kontext der Landwirtschaft sind die Tiertransporte. Diese sind infolge der Einführung von Exportprämien 1989 sprunghaft angestiegen. Die fortschreitende Zentralisierung der Schlachthöfe führt zu einer deutlichen Zunahme von Tiertransporten. Täglich werden Millionen von Tieren durch Europa oder gar interkontinental verfrachtet, was mit erheblichem Leid verbunden ist. Aus unterneh-

3 Immerhin hat es im August 2018 dazu geführt, dass ein Landwirt aus dem Emsland, der sein Rind auf dem Weg zum Schlachter willkürlich mit einem Stock direkt aufs Auge geprügelt hat, 2100 Euro Strafe zahlen musste (Anke Fritz 2019). 
merischer Perspektive des Einzelnen mag es ein „vernünftiger Grund“ im Sinne des deutschen Tierschutzgesetzes sein, dem ökonomischen Druck für solche Tiertransporte nachzugeben, aus der Perspektive einer ernst zu nehmenden Tierethik ist die gegenwärtige Praxis jedoch kaum rechtfertigungsfähig.

Der extrem hohe Produktivitätsdruck erzeugt beispielsweise auch in den Großbetrieben von Legehennen erhebliche Probleme mit den tierethischen Ansprüchen: Die engen Käfige oder Boxen lassen den Tieren kaum Bewegungsfreiheit, oft auch keine Möglichkeit, ihre Eier in einen gesonderten Bereich abzulegen. Ca. 50 Prozent der Hennen haben mindestens eine Knochenfraktur, weil ihre Ressourcen statt in stabile Knochen nur in die extrem hochgezüchtete Eierproduktion fließen (ca. 300 Eier pro Jahr, die Schalen brauchen Kalzium, das dann für den Knochenaufbau fehlt). Jährlich werden in Deutschland bis zu 45 Millionen männliche Küken vergast oder geschreddert und als Biomüll entsorgt, weil sie Züchtungslinien entstammen, die aufs Eierlegen ausgerichtet sind und man den männlichen Nachwuchs folglich nicht brauchen kann. Diskutiert wird, ob es Auswege aus diesem Missstand gibt, z. B. durch endokrinologische Verfahren oder Lasertechnik, mit deren Hilfe man das Geschlecht bereits im Ei bestimmen könnte. ${ }^{4}$ Für die relative Verbesserung der Praxis wäre es vorrangig, die getöteten männlichen Küken wenigstens stärker als Tierfutter zu verwerten sowie vor allem die Züchtung offener zu gestalten, so dass die Hühner je nach Geschlecht entweder für das Eierlegen oder für den Fleischverzehr Verwendung finden könnten.

\subsection{Tierwohl als ethischer Leitbegriff}

Seit einigen Jahren hat der Begriff „Tierwohl“ den zuvor üblichen ethischen Leitbegriff der „Artgerechtigkeit“ abgelöst (vgl. zum Folgenden Johann Ach 2018 sowie Initiative Tierwohl 2019). Es handelt sich um eine

4 Die Mehrkosten sind gering (beim Laserverfahren nicht mehr als ein Cent pro Ei), aber es gibt erhebliche Anwendungshürden (z. B. hohe Fehlerquoten) und das Grundproblem, dass die Küken nicht brauchbar sind, bleibt. Der Hauptvorteil ist, dass man die Küken früher, also in einem weniger schmerzempfindlichen Stadium töten kann (und für die Agrarbetriebe, dass sie sich einige Zeit der Bebrütung sparen). Vgl. https://www.bmel.de/DE/Tier/Tierwohl/_texte/Tierwohl-Forschung-In-O vo.html (abgerufen am 24.09.2019); über den aktuellen Stand der Züchtung von Zweinutzungsrassen informiert die Seite www.oekotierzucht.de/ueber-uns/ (abgerufen am 24.09.2019). 
utilitaristische, auf Leidvermeidung bezogene Kategorie, während „artgerechte Tierhaltung" eher auf ethologische Forschung rekurrierte. Beide Begriffe haben Stärken und Schwächen, aber in der öffentlichen Kommunikation kommt der euphemistische Begriff „Tierwohl“ besser an. Es klingt besser, wenn man nicht von Missständen spricht, sondern von Faktoren für das Wohlbefinden wie z. B. von Tiergesundheit, Hygiene und einem guten Stallklima, der Größe und räumlichen Strukturierung der Käfige bzw. Boxen oder den Möglichkeiten, den natürlichen Verhaltensweisen nachzugehen. Faktisch wird als Kriterium des Tierwohls weitgehend auf die Standards der „Artgerechtigkeit“ zurückgegriffen.

Die Grundlage für viele Bewertungssysteme des Tierwohls bildet das Konzept der „Fünf Freiheiten“: Die Tiere sollen frei von Hunger und Durst, von Schmerz, von Verletzungen, von Krankheiten sowie von Angst und Stress sein.

Maßnahmen für Tierwohl sind beispielsweise (Initiative Tierwohl 2019):

- Staubbaden: Geflügel „badet“ gern in der Bodeneinstreu zur Körperpflege, es ist ein sogenanntes Komfortverhalten. Anschließend ordnen die Tiere das Gefieder wieder mit dem Schnabel.

- Raufutter: Mit diesem Begriff ist Futter mit einem hohen Rohfasergehalt gemeint, wie Heu oder Stroh. Es dient nicht nur zur Sättigung, sondern auch als Beschäftigungsmaterial beim Bekauen und Zerwühlen.

- Antibiotikamonitoring: Die lückenlose Dokumentation aller den Tieren verabreichten Antibiotika.

- Fußballengesundheit: Die Füße und Fußballen von Hühnchen und Puten sind sehr sensible Körperstellen, die sich leicht entzünden können. Für das Wohlbefinden des Tieres ist es deshalb wichtig, dass diese gesund sind, was z. B. durch Einstreu für den Stallboden gefördert werden kann.

- Abschaffung des Schnabelkürzens bei Legehennen: Damit der spitze Hühnerschnabel nicht zur Waffe wird, war in der Legehennenhaltung lange Zeit das Kupieren der Schnäbel üblich. Seit Anfang 2017 ist das Schnabelkürzen aus Tierschutzgründen auch in konventionell geführten Betrieben verboten.

- Schweine müssen zwischen Funktionsbereichen (z. B. Fressen und Schlafen) wählen können. Nestbaumaterial aus langfaserigen, organischen Materialien muss in ständiger Reichweite der Sau sein. Das Absetzen darf nach frühestens 25 Tagen Säugezeit erfolgen. Der Ausstieg aus dem 
Schwanzkupieren muss „beschleunigt“ werden, die betäubungslose Ferkelkastration ist nicht erlaubt.

\subsection{Initiativen zur Umsetzung von Tierwohl}

Zum Tierwohl gibt es in Deutschland sowohl eine Brancheninitiative wie ein staatliches Label. In Österreich gibt es im Rahmen von „Bio Austria“ ähnliche Maßnahmen. Die Bio Austria vertritt etwa 60 Prozent der 21.000 Biobauern Österreichs mit einem Gesamtumsatz von etwa 300 Millionen Euro und ist damit einer der größten Bio-Verbände der EU. Bereits 1979 haben sich die Biobauern in Österreich zusammengeschlossen. In der Schweiz sind strengere Standards für Tierschutz gesetzlich verankert und werden regelmäßig kontrolliert, wobei eher der Begriff „Tiergerechtigkeit“ als „Tierwohl“ dominiert.

Die deutsche, bundesweite Brancheninitiative Tierwohl startete 2015 als Bündnis aus Lebensmitteleinzelhandel sowie Schlacht- und Landwirtschaftsbetrieben (Initiative Tierwohl 2019). Finanziert wird sie vom teilnehmenden Lebensmitteleinzelhandel. Die Unternehmen führen pro verkauftem Kilogramm Schweine- und Geflügelfleisch und -wurst derzeit (2019) 6,25 Cent an die Initiative ab. Mit diesem Geld (insgesamt derzeit ca. 130 Millionen Euro im Jahr) werden Landwirtinnen und Landwirte für die Umsetzung von Tierwohlmaßnahmen honoriert. ${ }^{5} \mathrm{Zu}$ den Grundanforderungen gehören beispielsweise für Schweinehalter der Stallklima- und der Tränkewassercheck. Speziell geschulte Fachexperten führen diese Checks durch. Sie untersuchen alle Lüftungssysteme der Stallanlage und beurteilen die Luftqualität. Auch Tageslicht muss garantiert werden. Das Tränkewasser wird nach festgelegten chemischen und mikrobiologischen Parametern beprobt. Um teilnehmen zu können, müssen Betriebe den Antibiotikaverbrauch ihrer Tiere analysieren lassen. Zusätzlich müssen sie sich für mindestens eine weitere Maßnahme entscheiden (z. B. zehn Prozent mehr Platz als gesetzlich vorgeschrieben oder ständigen Zugang zu Raufutter). Das System lässt flexibel unterschiedliche Maßnahmen zu, um den Möglichkeiten der Betriebe Rechnung zu tragen.

5 Jede teilnehmende Landwirtin und jeder teilnehmende Landwirt muss bestimmte Grundanforderungen umsetzen. Zusätzlich gibt es Wahlkriterien. Über ein Gütesiegel kann sich jeder Kunde beteiligen. 535 Millionen Tiere in 6670 Betrieben profitieren von der Initiative Tierwohl (August 2019). 
Neben der Anerkennung für die relativen Verbesserungen gibt es in den Medien auch heftige Kritik an der Initiative, da sie lediglich eine Oberflächenkorrektur zur Aufpolierung des Images sei, die grundlegenden Missstände der Tiermast und der aggressiven Preispolitik der Supermarktketten jedoch in keiner Weise ändere. So etwa Katrin Zeug:

„Es ist eine interessante Logik, die die Initiative Tierwohl vertritt: Wer den gequälten Masttieren in Deutschland zu einem besseren Leben verhelfen möchte, der solle Fleisch kaufen. Und zwar bei Aldi, Lidl, Kaufland, Rewe, Penny, Edeka, Netto oder Kaiser's Tengelmann. ,Der Kauf dieser Produkte', so heißt es auf der Internetseite von Tierwohl, trage dazu bei, ,unser gemeinsames Ziel zu erreichen: die Standards in der Nutztierhaltung in Deutschland aktiv, flächendeckend und Schritt für Schritt weiter zu verbessern““ (Katrin Zeug 2015).

Es wird kritisiert, dass die Initiative den Tieren nur marginale Verbesserungen bringe und sie vor allem auf eine Beruhigung des Gewissens der Verbraucher ziele, damit diese weiterhin das Pfund Fleisch für zwei Euro kaufen.

„So lange die gesetzlichen Standards so sind wie sie sind, wird der Wettbewerb immer auf Kosten der Tiere gehen. Bei der Initiative geht es mehr ums Image und darum, Gesetze zu verhindern, als um eine nachhaltige Verbesserung“ (Luise Molling von Foodwatch, zitiert nach Rosina Samtpfote 2016).

Trotz aller Kritik sind ethische Produktkennzeichnungen ein wichtiges Instrument der Aufklärung, Bewusstseinsbildung und Beteiligung der Verbraucher, damit sie - anstatt zu verdrängen - durch ihr Kaufverhalten dem Wunsch nach bestimmten ethischen Standards im Umgang mit Tieren Ausdruck verleihen. Zu vermeiden ist jedoch, dass das Vorhandensein freiwilliger Standards als Rechtfertigung für niedrigere gesetzliche Vorgaben dient.

\subsection{Das staatliche Tierwohllabel}

Das Bundesministerium für Ernährung und Landwirtschaft (BMEL) plant ein staatliches Tierwohllabel, wofür im Januar 2019 ein dreistufiges Verfahren eingeleitet wurde. Kriterien wurden zunächst für Schweine erarbeitet.

Letztlich bleibt es jedoch trotz mancher Verschärfungen der Vorschriften bei eher marginalen Verbesserungen und die Ursachenebene der massiven Fehlentwicklungen kommt kaum in den Blick. Diese liegt in der ex- 
tremen Maximierungslogik, die jeweils eine spezifische Funktion (z. B. Eierlegen, Fleischansatz oder Milchproduktion) bis an die Grenzen des Möglichen steigert und so nicht nur Gesundheit und Wohlbefinden der Tiere systemisch destabilisiert, sondern auch ein hohes Maß an nicht verwertbarem „Tiermüll“ erzeugt (z. B. auch die männlichen Kälber der auf Milchleistung hochgezüchteten Kühe). Das BMEL formuliert das Defizit offen:

„Ein oft vernachlässigter Aspekt ist die Genetik bzw. Zucht. So hat beispielsweise die Zucht auf hohe Milchleistung und leichte Melkbarkeit zu schwächeren Schließmuskeln und kürzeren Strichkanälen geführt und damit die Euterentzündung zu einer ,Berufskrankheit‘ von Milchkühen gemacht“ (BMEL 2019 b).

Es gilt ethisch zu klären, ab welcher Schwelle traditionelle oder gentechnische Tierzüchtung als Herabwürdigung der Kreatur zum reinen Produktionsfaktor gewertet werden muss. Es ist offensichtlich, dass die gegenwärtige Praxis oft nicht mit dem vereinbar ist, was sich vernünftiger Weise als das gesetzlich definierte „Tierwohl“ interpretieren lässt. Der Eigenwert der Tiere als Mitgeschöpfe wird massiv missachtet. Die segmentierte Logik der extremen monofunktionalen Steigerung einzelner Tierleistungen (z. B. Milch bei Kühen, Fleischansatz an der Brust bei Puten oder Eierproduktion bei Hennen) führt schon heute zu Nutztierhaltungssystemen, die Gesundheit und Wohlbefinden der Tiere ständig gefährden und darüber hinaus in ihrer Gesamtheit höchst instabil sind.

\subsection{Internationale Perspektiven}

Im internationalen Vergleich sind die deutschen, österreichischen und schweizerischen Tierschutzstandards relativ hoch. Allmählich wächst trotz der gravierenden Konflikte in Einzelfragen das Verständnis zwischen Tierrechtlern und landwirtschaftlichen Praktikern, u. a. weil eine artgerechte Haltung auch im Blick auf die Tiergesundheit, auf die Qualität des Fleisches sowie auf die Akzeptanz der Kunden erhebliche Vorteile bietet. Dennoch bestehen erhebliche Defizite sowohl auf der Gesetzgebungsebene als auch in der Umsetzung. Priorität muss die Verringerung der Vollzugsdefizite sowie die Vermeidung eines Wettbewerbs der Unterbietung tierethischer Standards in der internationalen Konkurrenz haben. Politisch bedeutet dies, dass Tierschutz als anerkannter Teil der christlichen und europäischen Kulturordnung stärker in den EU- und WTO-Richtlinien zu veran- 
kern ist. Er sollte als grundsätzliches Ziel neben Belangen wie „gleiche Wettbewerbsbedingungen" und „freier Warenverkehr" formuliert werden.

Das Umfassende Wirtschafts- und Handelsabkommen EU-Kanada (CETA: Comprehensive Economic and Trade Agreement) sowie das MercosurAbkommen in und mit den südamerikanischen Staaten und andere Freihandelsverträge verstärken in der gegenwärtigen Form das Tierschutzdumping erheblich.

Die Grundaussage des Weltagrarberichtes von 2009, dass kleinbäuerliche Strukturen im Blick auf Hungerbekämpfung, ökologische Verträglichkeit der Landwirtschaft sowie auf soziale Funktionen (Arbeit, Erhalt kultureller Bräuche [International Assessment of Agricultural Knowledge, Science and Technology for Development 2009, bes. 4-22]) oft deutlich besser abschneiden als die auf Export ausgerichteten Großplantagen, sollte um die Wertschätzung der positiven Effekte kleinbäuerlicher Strukturen für mehr Tierwohl ergänzt werden.

\section{Erhalt der Artenvielfalt: auch ein Thema für die Landwirtschaft}

\subsection{Ein Viertel der Arten ist vom Aussterben bedroht}

Die Verdrängung von Wildtieren durch die gegenwärtigen Formen der globalen Landnutzung ist unter sozialethisch-systemischen Gesichtspunkten kaum weniger problematisch als die unmittelbare Qual von Tieren in der Nutztierhaltung. Die massive Reduktion von Artenvielfalt und WildtierPopulationen, die als das sechste Massensterben der Evolutionsgeschichte bezeichnet wird (Ulrich Kutschera 2016, 57-66; Helmholtz-Zentrum für Umweltforschung [Hg.] 2019, 9), ist in wesentlichen Aspekten eine nicht intendierte Nebenwirkung der modernen Landwirtschaft. Auch wenn man in der ethischen Bewertung durchaus einen Unterschied zwischen unmittelbaren und indirekten Handlungsfolgen berücksichtigen sollte, sind gerade letztere typisch für die späte Moderne und aufgrund ihres systemischen Charakters von ganz eigenem Gewicht, das mit besonderer Dringlichkeit zu einem neuen Verantwortungsbewusstsein sowie einer Reflexion der Zurechenbarkeit von Rechenschaftspflichten auffordert (Markus Vogt ${ }^{32013,374-454) .}$

Der Verlust an Biodiversität muss neben und in enger Verbindung mit dem Klimawandel als das zweite große Problemfeld des gegenwärtigen Umweltwandels eingestuft werden. Die weltweiten Daten zu den globalen Funktionskreisläufen der ökologischen Systeme im Rahmen der PlanetaryBoundaries-Forschung zeigen, dass die Beeinträchtigung der Artenvielfalt 
bereits heute zu den zentralen kritischen Faktoren für den Erhalt der Lebensräume zählt (Will Steffen et al. 2015). Ein häufig unterschätzter Faktor ist der enge Zusammenhang von Biodiversität und Bodenfruchtbarkeit (Markus Vogt 2018, 265-275).

„Menschliche Aktivitäten sorgen dafür, dass heute mehr Arten vom Aussterben bedroht sind als jemals zuvor. Circa 25 Prozent der Arten in den meisten Tier- und Pflanzengruppen, also bis zu 1 Million Arten, sind bereits vom Aussterben bedroht. Viele wird das innerhalb der nächsten Jahrzehnte betreffen - es sei denn, es werden Maßnahmen ergriffen, um die Intensität der Treiber zu verringern, die für den Verlust der biologischen Vielfalt verantwortlich sind“ (Helmholtz-Zentrum für Umweltforschung [Hg.] 2019, 8).

Von den 1,75 Millionen wissenschaftlich bekannten Arten gelten 23 Prozent der Wirbeltiere (12 Prozent der Säugetiere, 61 Prozent der Reptilien, 31 Prozent der Amphibien, 46 Prozent der Fische) und 57 Prozent der Nichtwirbeltiere (73 Prozent der Insekten, 45 Prozent der Weichtiere, 86 Prozent der Krustentiere) als gefährdet (Eva Berié/Heide Kobert [Hg.] 2006, 726; auch IPBES 2019). Jährlich verschwinden bis zu 35.000 Arten. Wesentliche Ursachen sind die Beeinträchtigung oder Vernichtung von Lebensräumen, die Übernutzung von Ökosystemen, die Einführung fremder Arten, die allgemeine Umweltverschmutzung sowie die globale Erwärmung. Lange stand der isolierte Schutz einzelner Arten im Vordergrund, heute dagegen richtet sich der Fokus auf ökologische Gesamtzusammenhänge.

Viele Tierarten sind für die Erhaltung der pflanzlichen Biodiversität von grundlegender und in ihrer Vielfalt nicht substituierbarer Bedeutung (IPBES 2019 sowie Helmholtz-Zentrum für Umweltforschung [Hg.] 2019). Besonders dramatisch ist das Insektensterben. Die 2017 veröffentlichte „Krefelder Studie“ diagnostiziert eine Abnahme der Biomasse der Insekten seit 1990 um drei Viertel: „Our analysis estimates a seasonal decline of $76 \%$, and mid-summer decline of $82 \%$ in flying insect biomass over the 27 years of study" (Caspar A. Hallmann et al. 2017, 1). Die Studie gilt als wissenschaftlich solide und von weitreichender Bedeutung für den besorgniserregenden Zustand der Biodiversität in Deutschland und weltweit, insbesondere auf landwirtschaftlich genutzten Flächen. Dabei darf die Abnahme der Biomasse allerdings nicht mit "Artensterben“ gleichgesetzt werden: Die Quote der vom Aussterben bedrohten Insektenarten wird mit zehn Prozent berechnet (Helmholtz-Zentrum für Umweltforschung [Hg.] 
2019, 9). ${ }^{6}$ Dennoch ist dies für die Koevolution des Lebens und auch für die gesamte Landwirtschaft von kaum zu überschätzender Bedeutung. Das bayerische Volksbegehren „Rettet die Bienen“, an dem sich 1,8 Millionen Bürgerinnen und Bürger beteiligten und das im Juli 2019 vom Bayerischen Landtag als Gesetz angenommen wurde, ist Ausdruck eines beginnenden Bewusstseinswandels in der Bevölkerung auch im Blick auf Biodiversität. Die Entwicklung ethisch-systematischer Reflexionen hierzu steht jedoch erst am Anfang.

\subsection{Die expansive Landwirtschaft als Treiber}

Die expansive Landnutzung ist der entscheidende Treiber für den Verlust an Biodiversität. ${ }^{7}$ Seit vielen Jahren schreiten massive Brandrodungen der tropischen Wälder, in denen der größte Reichtum an Arten lebt, scheinbar unaufhaltsam voran. Nicht selten gibt es dabei ökologische Zielkonflikte, z. B. wenn der Urwald für Palmöl gerodet wird. Völlig zu Recht ist der Amazonas ins Blickfeld der Weltöffentlichkeit gerückt. Bolsonaros Vorhaltung, dass das im Rahmen des G7-Gipfels in Biarritz (August 2019) geäußerte Schutzinteresse kolonialistisch sei, ist entgegenzuhalten, dass die Rodungen - oft im Interesse internationaler Agrarkonzerne - in weit höherem Maße kolonialistisch und indigene Bevölkerungen die Leidtragenden sind. Flächengewinn für Rinderherden und Futtermittelproduktion ist das Hauptmotiv für die Rodung des Urwaldes. Der Konflikt zeigt, dass Agrarpolitik weltweit dabei ist, zu einem entscheidenden Machtfaktor aufzusteigen. Oft gibt es ein hartes Ringen um die Begrenzung der Agrarflächen durch Schutzgebiete. ${ }^{8}$

6 Da ein großer Teil der Insektenarten nicht wissenschaftlich klassifiziert ist, sind die Hochrechnungen hierzu von einigen Unsicherheiten geprägt.

7 So nimmt die Tierhaltung beispielsweise in der EU 70 Prozent der Fläche in Anspruch und ist darüber hinaus durch Futtermittelimport (etwa aus Lateinamerika) für viele Landnutzungsänderungen im Globalen Süden wesentlich mitverantwortlich (Antonia Weishaupt 2019, 125). Durch die Treibhausgase Kohlendioxid, Methan und Lachgas verantwortet die Tierhaltung in der EU ca. ein Achtel der Treibhausgasemissionen (Antonia Weishaupt 2019, 125).

8 Von großer Bedeutung für den Artenschutz sind Schutzgebiete, in denen menschliche Tätigkeiten wie Land- und Forstwirtschaft sowie Siedlungen eingeschränkt werden. Weltweit gibt es über 100.000 Schutzgebiete mit insgesamt knapp 20 Mio. $\mathrm{km}^{2}$ Fläche (inklusive Meeresflächen). Deutschland zählt 14 Nationalparks, die bisher allerdings noch keine einheitlichen Qualitätskriterien besitzen. 
Während der Bestand an Wildtieren hinsichtlich Zahl und Arten rapide zurückgeht, nimmt derjenige an Nutztieren zu: Derzeit gibt es deutlich mehr Biomasse Rind als Biomasse Mensch (600 Millionen Tonnen zu 400 Millionen Tonnen). Die globale Geflügelhaltung umfasst fast zehnmal so viele Individuen wie die Menschheit (68 Mrd. Tiere). Allein in Deutschland werden jährlich ca. 26 Millionen Schweine geschlachtet. Insgesamt werden jährlich mehr als acht Millionen Tonnen Fleisch geschlachtet (Albert Schweitzer Stiftung für unsere Mitwelt 2017). Die Futtermittelproduktion sowie der Bedarf an Weideflächen setzen den Planeten enorm unter Druck. Die Dynamik der Übernutzung natürlicher Ressourcen durch die globale Bevölkerungsentwicklung wird quantitativ durch die Steigerung der Nutztierhaltung weit übertroffen. ${ }^{9}$

\subsection{Vom Arten-zum Biodiversitätsschutz}

Artenschutz bezeichnet die Summe aller rechtlichen und planerischen Maßnahmen zum direkten Schutz einzelner Tier- und Pflanzenarten. Wichtigstes Instrument zur Bestimmung des Gefährdungsgrades von Arten und der Prioritäten des Schutzes waren lange die Red Data Books („Rote Listen“) der International Union for Conservation of Nature and Natural Resources (IUCN). Als ethisch-politische Leitkategorie für Artenschutz hat sich inzwischen der Begriff „Biodiversität“ durchgesetzt. Dieser meint nicht nur die Anzahl unterschiedlicher Arten, sondern ebenso die Vielfalt der Ökosysteme. Hierfür werden die Daten weltweiter Forschungen unter dem Dach der „Intergovernmental Platform on Biodiversity and Ecosystem Services (IPBES)“ zusammengefasst (IPBES 2019).

Wie lässt sich eine moralische Pflicht zum Schutz der Artenvielfalt begründen? Die lange übliche Begründung des Artenschutzes, dass Vielfalt eine notwendige Bedingung für ökologische Stabilität sei, wird nicht mehr geteilt, weil der Zusammenhang nicht zwingend und keinesfalls linear zu begreifen ist (Sachverständigenrat für Umweltfragen 1994, 71-73). Bei der Weltkonferenz für Biodiversität im Mai 2008 in Bonn dominierten ökonomische Argumentationen (TEEB 2010). Diese weisen zwei Stärken auf. Sie vermeiden die Grundsatzdebatte um den Eigenwert der Arten, die von Vertretern physiozentrischer Ansätze oft sehr apodiktisch postuliert wird und zu pola-

9 In der ethischen Reflexion münden solche Überlegungen in Fragen der Konsumethik, die hier nicht vertieft werden sollen. Vgl. dazu die Beiträge von Michael Rosenberger, Kurt Remele und Martina Besler in diesem Band. 
risierten, von der Praxis entkoppelten Kontroversen geführt hat. Außerdem packen sie die Akteure bei ihrem (langfristigen und aufgeklärten) Eigeninteresse, was stets eine starke Motivation ist. Es gibt jedoch auch offensichtliche Schwächen einer rein ökonomischen Begründung: Wir neigen dazu, nur die Arten zu schützen, für die wir ökonomische Verwertungsinteressen im Blick haben oder emotionale Verbundenheit empfinden. Dies ist bereits funktional ungenügend, weil damit ökologische Zusammenhänge zu wenig Beachtung finden. Das derzeit umfangreichste Konzept zur Erfassung von Ökosystemdienstleistungen ist die Studie „The Economics of Ecosystems and Biodiversity“ (TEEB 2010). Trotz aller Anstrengung bleibt es jedoch methodisch extrem schwierig, die komplexen Wirkungszusammenhänge mit monetären Werten zu beziffern und angemessen zu gewichten.

Auf der Basis einer Hermeneutik der biblischen Wabrnehmung von Tieren ergibt sich nach Wustmans eine Akzentverschiebung der Tierethik von der Leitmaxime der Leidvermeidung einzelner Tierindividuen hin zur Beachtung übergreifender ökologisch-schöpfungstheologischer Zusammenhänge (Clemens Wustmans 2015, 12, 98-101). Diese Perspektive verdeutlicht den Wert von biologischer Vielfalt als ethisches Gut auch jenseits von funktionalen, ökonomischen oder pathozentrischen Argumentationsmustern. Als Leitbegriff hierfür schlagen Hardmeier und Ott Kohabitation vor (Christof Hardmeier/Konrad Ott 2015, 100, 123, 148-149, 225). Dies scheint mir eine durchaus beachtenswerte Ergänzung zu den ganz auf die Maxime der Leidvermeidung von Tierindividuen zielenden utilitaristischen Ansätzen, die seit Peter Singers Animal Liberation dominieren. Schöpfungstheologisch haben wir auch eine Verantwortung für den Bestand der Arten im gemeinsamen „Haus der Schöpfung“. Deren akute Bedrohung durch die gegenwärtigen Formen von Landwirtschaft ist ein in der öffentlichen Debatte bisher nicht adäquat bewusster Aspekt. Für eine Kultur des respektvollen Umgangs mit Tieren wird es vor allem darum gehen, die indirekten, für die meisten unsichtbaren Auswirkungen des menschlichen Handelns auf die Lebensbedingungen von Tieren bewusst zu machen.

Es ist Aufgabe der christlichen Ethik, die in der öffentlichen Debatte und nicht selten auch in Gesetzestexten in Anspruch genommenen theologisch-ethischen Begriffe mit verbindlichem Inhalt für die aktuellen Probleme der Tierethik zu füllen und dabei interdisziplinär über Instrumente der Umsetzung zu diskutieren. So läge es beispielsweise nahe, einen Emissionshandel für den hohen Anteil an Treibhausgasen aus der Landwirtschaft und insbesondere der Tierhaltung einzuführen (Antonia Weishaupt 2019, 73-99). Eine flächenbezogene Definition maximal zulässiger Treibhausgasemissionen würde weiterhelfen (Antonia Weishaupt 2019, 99-110). 


\section{Was ist die theologisch-ethische Kompetenz in der Debatte?}

In der Fachdebatte um Tierwohl und artgerechte Tierhaltung sind Theologinnen und Theologen vor allem Lernende. Die extreme Tiervergessenheit ist in der von christlichen Wurzeln geprägten Zivilisation entstanden und sollte von daher nicht mit dem Blick einer vermeintlich überlegenen abstrakten Moral kritisiert werden. Sie sollte vielmehr in eine Auseinandersetzung auch mit den Ambivalenzen der eigenen Traditionen und Geisteshaltungen münden (Simone Horstmann et al. 2018). Tierschutz ist auch in der Enzyklika Laudato si' eher ein Randthema, wenngleich der Text einen durchaus sehr prägnanten und innovativen schöpfungstheologischen und ethischen Rahmen für eine christliche Tierschutzethik liefert. Dabei ist jedoch zu beachten, dass der kirchenamtliche Text teilweise paränetischen Charakter hat und eine ethische Systematik nicht ersetzen kann. Insgesamt besteht in der Tierschutzethik von kirchlicher und theologischer Seite trotz einiger früher und prägnanter Beiträge erheblicher Nachholbedarf, der erst in jüngerer Zeit mit breiterer Resonanz verringert wird (vgl. dazu ohne Anspruch auf Vollständigkeit: Rainer Hagencord 2011; Traugott Jähnichen/Clemens Wustmans [Hg.] 2012; Amosinternational 2014; Michael Rosenberger 2015; Clemens Wustmans 2015; Kurt Remele 2016; Martin M. Lintner 2017; Simone Horstmann et al. 2018; MThZ 4/2019). Vor allem an einer sozialethischen Auseinandersetzung mit den Systembedingungen der Nutztierhaltung in der modernen Landwirtschaft sowie des damit verknüpften Verlustes von Biodiversität fehlt es immer noch erheblich. Tierschutz ist ein in der christlichen Ethik massiv vernachlässigtes Thema.

Gleichwohl können Theologie und Kirche durchaus Spezifisches in die Debatte einbringen. Ich möchte abschließend vier Aspekte nennen:

- Dialogpartner in der polarisierten Debatte zwischen Tier- und Umweltschützern auf der einen und Vertretern der Landwirtschaft und der Lebensmittelindustrie auf der anderen Seite: Wie explosiv diese Debatte ist, hat sich beispielsweise in jüngster Zeit beim bayerischen Volksbegehren zum Artenschutz gezeigt, das der Bauernverband lange zu blockieren suchte. Die Kirchen haben hier viel Vermittlungsarbeit geleistet, insbesondere Katholische Landvolk-, Landfrauen- und Landjugendverbände sowie evangelische und katholische Landvolkshochschulen. Auch auf der oberen Hierarchieebene ist das Thema Landwirtschaft seit den 1980er-Jahren in der Deutschen Bischofskonferenz (und vermutlich ähnlich in anderen Ländern) stark präsent (nicht zuletzt, weil Bischöfe überproportional aus der Landwirtschaft stammen). So hat es 
immer wieder intensive Auseinandersetzungen mit den Handlungsbedingungen und Zukunftsperspektiven der Landwirtschaft gegeben (vgl. beispielsweise EKD/DBK 2003), wobei der Tierschutz von kirchenamtlicher Seite jedoch nie systematisch im Mittelpunkt stand (eine Ausnahme ist die Studie: Evangelisch-Lutherische Kirche in Norddeutschland 2017).

- Ethisch-systematische und schöpfungstheologische Vertiefung der Debatte: Die Rezeption des theologischen Begriffs „Tiere als Mitgeschöpfe“ im deutschen Grundgesetz und im Tierschutzgesetz bzw. „Würde der Kreatur" in der Schweizer Verfassung ist bemerkenswert. Der Terminus steht stellvertretend für das Bewusstsein, dass es beim Tierschutz über die einzelnen Rechtsnormen, die der Staat erlassen kann, hinaus auch um Grundhaltungen geht. Soll der Begriff nicht zur Floskel und Leerformel werden, muss er mit einem für die plurale Gesellschaft verständlichen Inhalt gefüllt werden. Dafür bedarf es einer Übersetzung der Schöpfungstheologie in den Kontext der Tierethik und ihrer praktischen Herausforderungen. Es fehlt an einem hörbaren Widerspruch da$\mathrm{zu}$, dass die derzeit immer noch gesetzlich erlaubten Haltungsbedingungen für Nutztiere dem theologisch „entliehenen“ Leitbegriff Hohn sprechen. Eine nähere ethische Entfaltung des Konzepts der Kohabitation im Sinne der ethischen Orientierung an gesamtökologischen bzw. schöpfungstheologischen Zusammenhängen könnte eine wichtige Ergänzung zu der im Tierschutz dominierenden pathozentrischen Orientierung am einzelnen Tierwohl darstellen. Christliche Tierethik kann die Anliegen pragmatischer, pathozentrischer und ökosystemarer $\mathrm{Zu}$ gänge aus schöpfungstheologischer Sicht verknüpfen, indem sie Perspektiven einbringt, die sowohl über personalistische wie über rein funktionale Konzeptionen hinausweisen und die Schöpfung als eine Welt unaufhebbarer Konflikte, in der Kompromisse unvermeidlich sind, verstehen (Wilhelm Korff 1979). Damit kann sie auch auf ethischsystematischer Ebene eine wichtige Vermittlungsfunktion wahrnehmen.

- Ethos eines achtsamen Umgangs mit Tieren: Es gibt zahlreiche bäuerlichchristliche Traditionen, die in ländlichen Regionen immer noch lebendig sind und große Ausstrahlung auf das Bewusstsein der Menschen haben (z. B. in Bayern der Leonhardi-Ritt, für den überall Pferde aufs Prächtigste geschmückt werden; zu weiteren Beispielen vgl. Beatrice van Saan-Klein et al. [Hg.] 2004, 21-34; Franz-Theo Gottwald 2004, 73-124; Michael Rosenberger 2015; Martin M. Lintner 2020). Sie stellen eine wichtige kulturelle Alternative zu dem rein instrumentellen Umgang mit Tieren dar. Angesichts der vielerorts zurückgedrängten bäuer- 
lichen Tradition brauchen diese Bräuche eine bewusste Pflege, um erhalten zu bleiben. Es bietet sich an, diese für eine Vermittlung des neu erwachenden Bewusstseins für den tierethischen Gehalt biblischer Texte zu nutzen und dabei auch praktische Konsequenzen für einen veränderten Umgang mit Tieren im Alltag zu verdeutlichen. Ein Versuch der Wiederbelebung solcher Traditionen sind beispielsweise die auf dem Petersplatz seit 2009 wieder stattfindenden liturgischen Tiersegnungen. Auch die Tradition, am Freitag auf Fleischverzehr zu verzichten, war eine über Jahrhunderte wirksame Verankerung einer gewissen tierethischen Distanz zur Selbstverständlichkeit des Fleischverzehrs, die heute weitgehend ihre kulturelle Prägekraft verloren hat. Ein anderes Element christlicher Tradition, in dem sich eine empathische Wahrnehmung von Tieren ausdrückt, sind zahlreiche Heiligenlegenden: Tiere sind dort oft Symbole von Emotionen, Tugenden, Ängsten oder Naturkräften. Ähnlich wie in vielen Mythen und Märchen wird der Umgang mit Tieren als Spiegel der eigenen Seele dargestellt und poetisch ausgestaltet. Der entscheidende Beitrag der christlichen Tradition liegt hier nicht in begrifflichen Unterscheidungen, sondern in dem reichen Schatz an Narrativen und Traditionen für den „Traum von Frieden zwischen Mensch und Tier“" (Michael Rosenberger 2015), der über die biblischen Texte hinaus beispielsweise auch in diesen Heiligenlegenden sowie in der christlichen Kunst vielfältig Ausdruck gefunden hat.

- Internationale, interkulturelle und interreligiöse Dimension der Debatte: Das internationale Engagement der Kirchen, insbesondere der Hilfswerke, z. B. von Misereor für Kleinbauern in Entwicklungsländern und ihre Verteidigung gegen die großen Agrarkonzerne, ist für zahllose Menschen ein Zeichen der Hoffnung in einer oft aussichtslos erscheinenden Situation. ${ }^{10}$ Tierethik braucht eine entwicklungs- und sozialpolitische Flankierung. Hierbei gibt es zahlreiche Überschneidungen der Interessen der zahllosen Kleinbauern im Globalen Süden und der Anliegen des Tierschutzes. Dies ist eine starke Bestätigung für den ökosozialen Ansatz, wie er beispielsweise der Enzyklika Laudato si’ zugrunde liegt.

10 Eine deutliche Kritik der expansiven Landwirtschaft, die Boden für Weiden und Tierfutteranbau sowie Palmölplantagen beansprucht, findet sich beispielsweise im Abschlussdokument der Amazonien-Konferenz vom Oktober 2019; Bischofssynode - Sonderversammlung für Amazonien 2019. 


\section{Literatur}

Ach, Johann 2018, Tierwohl und Ethik, in: Ach, Johann/Borchers, Dagmar (Hg.) 2018, Handbuch Tierethik. Grundlagen - Kontexte - Perspektiven, Stuttgart, 208-212.

Ach, Johann/Borchers, Dagmar (Hg.) 2018, Handbuch Tierethik. Grundlagen Kontexte - Perspektiven, Stuttgart.

Albert Schweitzer Stiftung für unsere Mitwelt 2017, Schlachtzahlen 2016, https://al bert-schweitzer-stiftung.de/aktuell/schlachtzahlen-2016 (abgerufen am 24.09.2019).

Amosinternational 2014, Die Würde der Tiere ist antastbar, in: Internationale Zeitschrift für christliche Sozialethik 3/2014.

Berié, Eva/Kobert, Heide (Hg.) 2006, Der Fischer Weltalmanach 2007. Zahlen Daten - Fakten, Frankfurt a. M., 724-726.

Bischofssynode - Sonderversammlung für Amazonien 2019, Amazonien: Neue Wege für die Kirche und eine ganzheitliche Ökologie. Schlussdokument, hg. von Misereor et al., Aachen etc.

Bundesministerium für Ernährung und Landwirtschaft (BMEL) 2015, Tierschutzbericht der Bundesregierung 2015, Berlin.

Bundesministerium für Ernährung und Landwirtschaft (BMEL) 2019a, Grundzüge der Gemeinsamen Agrarpolitik (GAP) und ihrer Umsetzung in Deutschland, www.bmel.de/DE/Landwirtschaft/Agrarpolitik/_Texte/GAP-NationaleUmsetzu ng.html (abgerufen am 24.09.2019).

Bundesministerium für Ernährung und Landwirtschaft (BMEL) 2019 b, Die Tierwohl-Initiative des BMEL, https:/www.bmel.de/DE/Tier/Tierwohl/tierwohl_no de.html (abgerufen am 28.08.2019).

European Bird Census Council (EBBC) 2019, The EuroBirdPortal (EBP), Barcelona; https://www.ebcc.info/art-598/ (abgerufen am 19.09.2019).

Evangelische Kirche in Deutschland/Deutsche Bischofskonferenz (EKD/DBK) 2003, Neuorientierung für eine nachhaltige Landwirtschaft (Gemeinsame Texte 18), Hannover/Bonn.

Evangelisch-Lutherische Kirche in Norddeutschland 2017, Zwischen Landwirtschaft und Industrie - Diskussionshilfe zur Tierhaltung am Beispiel der Situation in Mecklenburg-Vorpommern, Kiel.

Franziskus 2015, Laudato si'. Enzyklika über die Sorge für das gemeinsame Haus (24.05.2015) (Verlautbarungen des Apostolischen Stuhls 202), Bonn.

Fritz, Anke 2019, Wegen Tierquälerei muss ein Landwirt 2.100 Euro Strafe zahlen, in: agrarheute vom 15.08.2019, https://www.agrarheute.com/management/recht/ landwirt-schlug-rind-2100-euro-geldstrafe-wegen-tierquaelerei-556868 (abgerufen am 24.09.2019).

Gottwald, Franz-Theo 2004, Geschöpfe wie wir: zur Verantwortung des Menschen für das Tier. Kirchliche Positionen, München.

Grimm, Herwig/Wild, Markus 2016, Tierethik zur Einführung, Hamburg. 
Haber, Wolfgang/Held, Martin/Vogt, Markus (Hg.) 2016, Die Welt im Anthropozän. Erkundungen im Spannungsfeld zwischen Ökologie und Humanität, München.

Hagencord, Rainer 2011, Die Würde der Tiere. Eine religiöse Wertschätzung, Gütersloh.

Hallmann, Caspar A./Sorg, Martin/Jongejans, Eelke/Siepel, Henk et al. 2017, More than 75 percent decline over 27 years in total flying insect biomass in protected areas, in: PLOS ONE 12 (10): e0185809; DOI: org/10.1371/journal.pone.0185809.

Hardmeier, Christof/Ott, Konrad 2015, Naturethik und biblische Schöpfungserzählungen. Ein diskurstheoretischer und narrativ-hermeneutischer Brückenschlag, Stuttgart.

Helmholtz-Zentrum für Umweltforschung (Hg.) 2019, Das „Globale Assessment“ des Weltbiodiversitätsrates IPBES, Leipzig, https://www.helmholtz.de/fileadmin /user_upload/IPBES-Factsheet.pdf (abgerufen am 19.09.2019).

Hörster, Norbert 2004, Haben Tiere eine Würde? Grundfragen der Tierethik, München.

Horstmann, Simone/Ruster, Thomas/Taxacher, Gregor 2018, Alles, was atmet. Eine Theologie der Tiere, Regensburg.

Initiative Tierwohl 2019, Webseite; https://initiative-tierwohl.de (abgerufen am 28.08.2019).

Intergovernmental Platform on Biodiversity and Ecosystem Services (IPBES) 2019, Global assessment report on biodiversity and ecosystem services. Summary for policymakers, https://ipbes.net/sites/default/files/ipbes_7_10_add.1_en_1.pdf (abgerufen am 19.09.2019).

International Assessment of Agricultural Knowledge, Science and Technology for Development (IAASTD)/Albrecht, Stephan/Engel, Albert (Hg.) 2009, Weltagrarbericht. Synthesebericht, Hamburg, http://hup.sub.uni-hamburg.de/volltexte/20 09/94/pdf/HamburgUP_IAASTD_Synthesebericht.pdf (abgerufen am 24.09.2019).

Jähnichen, Traugott/Wustmans, Clemens (Hg.) 2012, Tierethik. Biblisch-historische Grundlagen - normative Perspektiven - aktuelle Herausforderungen (Sozial-ethische Materialien Heft 2), Kamen.

Korff, Wilhelm 1979, Kernenergie und Moraltheologie. Der Beitrag theologischer Ethik zur Frage allgemeiner Kriterien ethischer Entscheidungsprozesse, Frankfurt a. M.

Kutschera, Ulrich 2016, Der Mensch im Anthropozän - Hat das sechste Massenaussterben bereits begonnen?, in: Haber, Wolfgang/Held, Martin/Vogt, Markus (Hg.) 2016, Die Welt im Anthropozän. Erkundungen im Spannungsfeld zwischen Ökologie und Humanität, München, 57-66.

Lintner, Martin M. 2017, Der Mensch und das liebe Vieh. Ethische Fragen im Umgang mit Tieren. Mit Beiträgen von Christoph J. Amor und Markus Moling, Innsbruck. 
Lintner, Martin M. 2020, Werte in der Landwirtschaft: Grundaspekte eines bäuerlichen Berufsethos, in: Müller, Sigrid/Höllinger, Stephanie/Baldt, Bettina 2020 (Hg.), Werte im Beruf. Ethik und Praxis im Gespräch (Studien der Moraltheologie. Neue Folge 14), Münster, 69-94.

Michel-Berger, Simon 2019 a, Kastenstandhaltung. Aufgestanden und gegangen, in: Wochenblatt vom 22.08.2019; https://www.wochenblatt-dlv.de/politik/aufges tanden-gegangen-557170 (abgerufen am 18.01.2021).

Michel-Berger, Simon 2019 b, Klimaschutz. Landwirtschaft und Ernährung ändern, in: Wochenblatt vom 13.08.2019; https:/www.wochenblatt-dlv.de/politik/ landwirtschaft-ernaehrung-aendern-556762 (abgerufen am 18.01.2021).

MThZ - Münchener Theologische Zeitschrift 2019, Theologie nach dem ,animal turn“. Themenheft 4/2019 (Jg. 70).

Nida-Rümelin, Julian 2005, Tierethik I: zu den philosophischen und ethischen Grundlagen des Tierschutzes, in: Nida-Rümelin, Julian (Hg.) 2005, Angewandte Ethik. Die Bereichsethiken und ihre theoretische Fundierung. Ein Handbuch, Stuttgart, 514-539.

Nussbaum, Martha 2010, Die Grenzen der Gerechtigkeit: Behinderung, Nationalität und Spezieszugehörigkeit, Frankfurt a. M.

Otterstedt, Carola/Rosenberger, Michael (Hg.) 2009, Gefährten - Konkurrenten Verwandte. Die Mensch-Tier-Beziehung im wissenschaftlichen Diskurs, Göttingen.

Oxford Center for Animal Ethics, http://www.oxfordanimalethics.com/home/ (abgerufen am 24.09.2019).

Precht, Richard David 2016, Tiere denken. Vom Recht der Tiere und den Grenzen des Menschen, München.

Remele, Kurt 2016, Die Würde des Tieres ist unantastbar. Eine neue christliche Tierethik, Kevelaer.

Röhrs, Manfred/Sambraus, Hans 1998, Tierhaltung, in: Korff, Wilhelm (Hg.) 1998, Lexikon der Bioethik, Bd. 3, Gütersloh, 539-554.

Rosenberger, Michael 2015, Der Traum vom Frieden zwischen Mensch und Tier. Eine christliche Tierethik, München.

van Saan-Klein, Beatrice/Dirscherl, Clemens/Vogt, Markus (Hg.) 2004, „... es soll nicht aufhören Saat und Ernte“. Ein Praxisbuch zum Mehrwert nachhaltiger Landwirtschaft, München.

Sachverständigenrat für Umweltfragen (SRU) 1994, Umweltgutachten 1994: für eine dauerhaft-umweltgerechte Entwicklung, Wiesbaden.

Samtpfote, Rosina 2016, Fleisch-Kennzeichnung - Initiative Tierwohl in der Kritik, http://www.rosinas-welt.de/samt-e-v-newsletter/fleisch-kennzeichnung-initiativetierwohl-in-der-kritik/ (abgerufen am 28.08.2019).

Sommer, Volker 2016, Planet ohne Affen? Zur Zukunft unserer Mitprimaten, in: Haber, Wolfgang/Held, Martin/Vogt, Markus (Hg.) 2016, Die Welt im Anthropozän. Erkundungen im Spannungsfeld zwischen Ökologie und Humanität, München, 67-78. 
Steffen, Will/Richardson, Katherine/Rockström, Johan/Cornell, Sarah Elisabeth et al. 2015, Planetary boundaries: Guiding human development on a changing planet, in: Science express 15 January 2015, 1-16.

The Economics of Ecosystems and Biodiversity (TEEB) 2010, Ecological and Economic Foundations, London/Washington.

Vogt, Markus ${ }^{3}$ 2013, Prinzip Nachhaltigkeit. Ein Entwurf aus theologisch-ethischer Perspektive, München.

Vogt, Markus/Ostheimer, Jochen/ Uekötter, Frank (Hg.) 2013, Wo steht die Umweltethik? Argumentationsmuster im Wandel (Beiträge zur sozialwissenschaftlichen Nachhaltigkeitsforschung 5), Marburg.

Vogt, Markus 2014, Zur moralischen Grammatik der Solidarität und ihrer (begrenzten) Anwendbarkeit auf intergenerationelle Konflikte, in: Jahrbuch für Ethik und Recht 22, 95-114.

Vogt, Markus 2018, Die Zehn Gebote des Bodenschutzes. Schöpfungsethische Leitlinien zum Umgang mit Land, in: Stimmen der Zeit 143, 265-275.

Vogt, Markus 2019, Tierethik. Philosophische und theologische Ansätze des gegenwärtigen „animal turn“, in: Münchener Theologische Zeitschrift 70, 333-354.

Weishaupt, Antonia 2019, Nachhaltigkeits-Governance tierischer Nahrungsmittel in der EU, Marburg.

World Wide Fund for Nature (WWF) 2012, Hintergrundinformation Rote Listen der bedrohten Tier- und Pflanzenarten, http://www.wwf.de/fileadmin/fm-wwf/P ublikationen-PDF/HG_Rote_Liste_10_10.pdf (abgerufen am 24.09.2019).

Wustmans, Clemens 2015, Tierethik als Ethik des Artenschutzes. Chancen und Grenzen, Stuttgart.

Zentralkomitee der deutschen Katholiken (ZdK) 2003, Agrarpolitik muss wieder Teil der Gesellschaftspolitik werden, Bonn.

Zeug, Katrin 2015, Initiative Tierwohl. Schweinerei im Kühlregal, in: https://www. spiegel.de/wirtschaft/initiative-tierwohl-schweinerei-im-kuehlregal-a-1053463.ht ml (abgerufen am 26.08.2019); ursprünglich in: enorm 4/2015: Generation grün. 